\title{
Integrated Transient Stability Analysis with Multi-Large-Scale Solar Photovoltaic in Distribution Network
}

\author{
Ngoc Thien Nam Tran', Hong-Tzer Yang ${ }^{1}$,Minh Quan Duong ${ }^{2 *}$ \\ ${ }^{1}$ National Cheng Kung University, Tainan, Taiwan \\ ${ }^{2}$ The University of Danang - University of Science and Technology, Danang, Vietnam \\ Received: October 30, 2020; Accepted: November 12, 2020
}

\begin{abstract}
Using renewable energy sources (RES) to face energy shortages in the context of rising load demand is a central issue in national power system planning. With low investment rates, rapid return on capital as well as many incentives from the government, solar energy is being more invested than other types of RES, as it can be seen as a rooftop and large-scale Photovoltaic (LSPV) system. However, if the LSPV penetrates too much into the grid, it will weaken the stability of the system, especially when a fault occurs. This paper aims to evaluate the transient stability of an actual distribution grid (DG) under different penetration levels of LSPV by ETAP software. The frequency and voltage responses will be presented in the results along with the rotor angle variation of a conventional generator located near the LSPV.
\end{abstract}

Keywords: Distribution grid, ETAP, LSPV, penetration level, transient stability

\section{Introduction}

The challenge of rising load demand coupled with regulations to reduce $\mathrm{CO}_{2}$ emissions is the main factor that makes renewable energy sources (RES) increasingly developed. The governments have issued policies to encourage and boost the RES installation to meet electricity demand in the future by prioritizing the purchase of electricity from RES, reducing construction taxes, etc. According to International Renewable Energy Agency (IRENA), 2019 witnessed a slowdown in building RES when the capacity expansion index was remarkably low. However, the total capacity expansion was still accounted for at least $70 \%$ of the global expansion, compared to 2018 [1]. Similarly, the renewable share of total generation capacity rose from $33.3 \%$ in 2018 to $34.7 \%$ in 2019 . Because of falling price and advanced technology, RES always maintains rapid growth with improved operating efficiency. The countries leading the current trend are concentrated in Asia such as China, India, Japan and Saudi Arabia [2]. Among RES, photovoltaic (PV) is the most developed thanks to many advantages such as low investment rates, rapid return on capital, noiselessness, flexible size, and less maintenance. Due to the growth of RES to cover the energy rising demand, there is an urgent need to analyze the impact of RES penetration in power systems and the development of ancillary services to improve the stability of systems.

Many studies have been conducted on PV with different problem approaches. Some researches

\footnotetext{
${ }^{*}$ Corresponding author: Tel.: (+84) 978.468.468

Email:dmquan@dut.udn.vn
}

demonstrated the benefit and detriment of PV penetration into the grid [3] when faults occur. In [4], the papers focus on optimizing power flow in the grid, finding optimal PV connection locations, or control solutions to improve operating efficiency, but the PV power level is assumed to be quite low in the grid or only care about the steady state of the grid. On the contrary, some of the cited works show that depending on the penetration level of PV and the condition of the load, it can cause a lot of voltage effects and many grid problems facing, offering necessary adjustment solutions, especially inverter control in PV [5]. The studies show that LSPV penetration causes a diverse impact on the grid in dynamic mode but the systems used in these studies are relatively small and LSPV focuses only on one connecting point. Meanwhile, LSPV plants are widely distributed in the gird. Similarly, the transient stability analysis is performed in [6] by using the IEEE standard system to consider the system behavior under fault conditions. The result shows that the system is failed to retain its stability at a higher level penetration of PV.

This paper evaluates the system transient stability with different levels of LSPV plants penetration based on the actual distribution grid (DG). The impacts of LSPV will be reviewed in detail about fault-ride through capability and the impact on nearby generators in the grid. The Quang Ngai distribution grid - Vietnam, which is chosen in this study, is a hot spot in Vietnam with many PV plants in the process of completing and connecting while these grid planning issues have not been proposed yet. The model will be simulated by ETAP software and UDM tool with various fault scenarios. 
The results have been presented the positive and negative impacts of LSPV plants when they are connected to the grid so that reasonable operation plans can be devised.

\section{Study case}

\subsection{Distribution grid model}

Quang Ngai DG is one of the important parts of Vietnam power system in the central, composed of 31 busbars, 53 transmission lines, 3 generating units and supplies to 21 loads with a total demand of approximately $760 \mathrm{MW}$. This grid has a full of main voltage levels of the transmission system in Vietnam, namely $500-220 \mathrm{kV}$ and $110 \mathrm{kV}$ distribution system along with conventional generators.

On the other hand, RES projects, especially solar energy, are planned in this area to ensure that the load plans are met due to the growing industrial zones. By using ETAP, the modeled grid with a single-line diagram is shown in Fig.1.

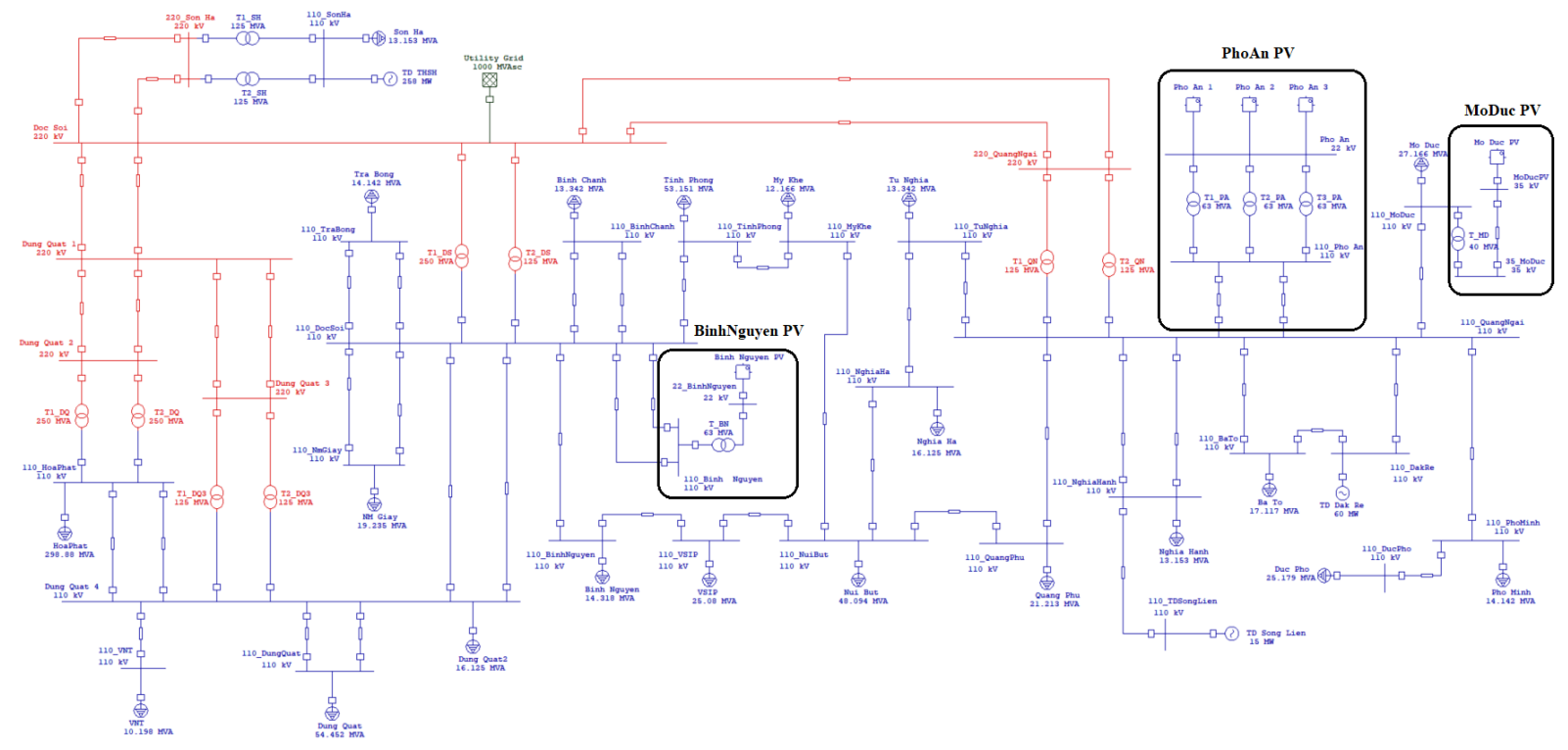

Fig. 1. Single line diagram of Quangngai distribution grid.

\subsection{Large-scale photovoltaic plants}

Based on the actual situation of the grid, electricity from conventional generators is insufficient to supply the local load. Therefore, the chance to penetrate RES becomes easy. Currently, 3 LSPV plants have been built and injected $210 \mathrm{MW}$ into the grid, responsive to $30 \%$ load demand and more LSPV projects are going to be accepted extension, following the planning power system of Vietnam. Specifically, three LSPV plants of this study are MoDuc PV, BinhNguyen PV and PhoAn PV, which have the same installed technology without energy storage system and plant information is presented in Table 1 .

Table 1. Information of LSPV plants

\begin{tabular}{|c|c|c|}
\hline Plants & $\begin{array}{c}\text { Capacity } \\
\text { (MW) }\end{array}$ & Connected Bus \\
\hline MoDuc PV & 19.2 & $110 \_$MoDuc \\
\hline BinhNguyen PV & 40.8 & 110_DocSoi \\
\hline PhoAn PV & 150 & 110_QuangNgai \\
\hline
\end{tabular}

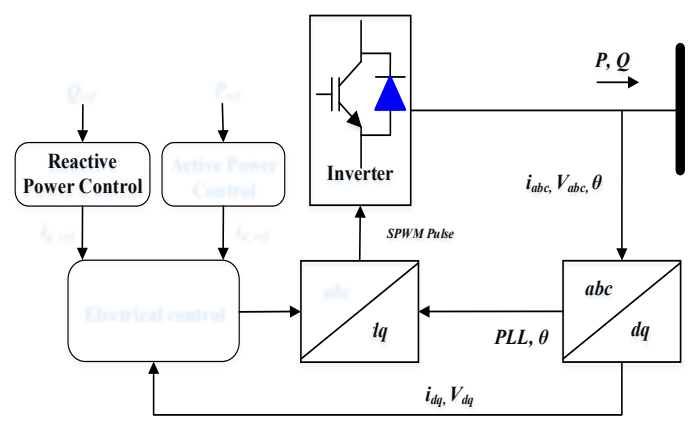

Fig. 2. The PQ control frame of full size inverter.

\subsection{Vietnam grid code for renewable energy}

In order to operate safely and stably, RES needs to comply with the grid code during connection. According to the requirements of the Vietnam grid [7], LSPV plants must follow two main points:

- Operating in the range of frequency and voltage as shown in Fig. 3. 
- During the fault and after clearing the fault, LSPV plants must remain connected to the grid if the voltage response overcomes the low voltage ridethrough (LVRT) in Fig.4.

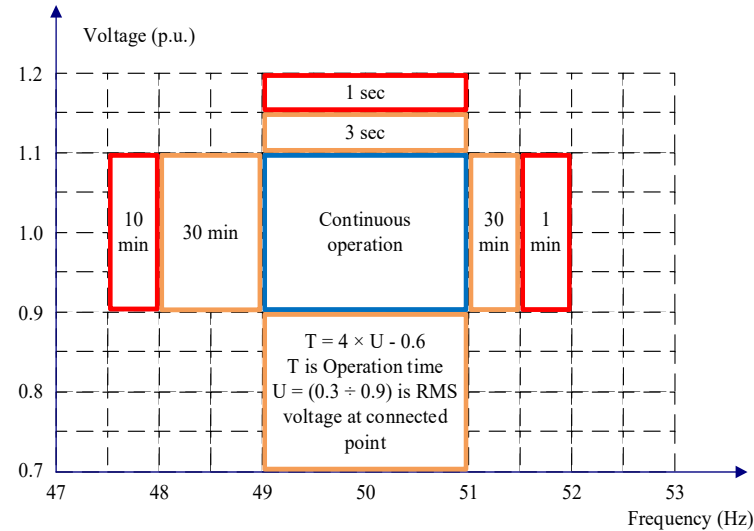

Fig. 3. Operation range for renewable energy.

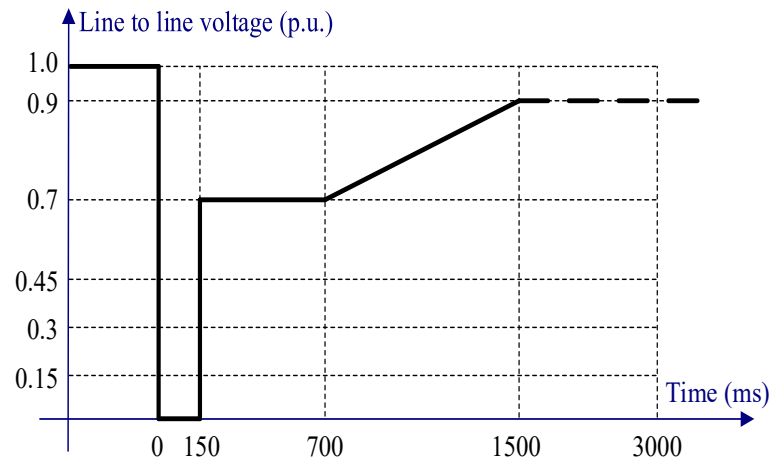

Fig. 4. The low voltage ride-through of grid code.

\section{Transient stability analysis and scenarios}

\subsection{Transient stability problem}

Stability of power system can be understood as the system ability to maintain and recover to a normal operating condition after being subjected to faults such as loss of generation, line outages and single line to ground fault, .... The transient stability problem, which focuses on evaluating the frequency and voltage response and impact to nearby conventional generators occurs when a failure with LSPV penetration, will be considered in this paper.

The increase in the connection of LSPV plants causes changes in the structure and operational characteristics of the system, namely the changes in voltage deviation angle and system inertia, which depends mostly on rotational inertia of generators, leading to higher potential risks of disturbances. While adding more PVs to the grid can increase the inertia, replacing other generators with PVs can make it decreased, which finally may cause a negative effect on the system's transient stability. The rotational inertia can control the frequency deviations and synchronization speed in the power system whenever there is a disturbance causing an imbalance between the input mechanical power and the output power of the generator. Thus, the available response time to react to events like short-circuit faults on line/busbar or generation outages is increased. This inertia can be determined through electromechanical dynamic behaviors governing by the swing equation. In this equation, the output power from the generator is described by a nonlinear function of the rotor angle [8]:

$$
J_{s} \frac{d^{2} \theta_{m}}{d t^{2}}=T_{i n}-T_{o u t}
$$

where: $T_{\text {in }}$ and $T_{\text {out }}$ are mechanical torque and electrical torque, respectively; $J_{s}$ is the total moment of inertia; $\theta_{m}$ represents the angular displacement of the rotor.

Multiplying by angular velocity $\omega$, equation (1) becomes:

$$
J_{s} \omega \frac{d^{2} \theta_{m}}{d t^{2}}=P_{i n}-P_{o u t}
$$

where: $P_{\text {in }}$ and $P_{\text {out }}$ refer to input mechanical power and output electrical power, respectively.

In the generator with $p$ poles, the relationship between electrical angle $\theta$ and mechanical angle $\theta_{m}$ is:

$$
\theta=\frac{p}{2} \theta_{m}
$$

From (2) and (3), with $M$ denoting inertia constant, $H$ is per unit inertia constant, $\omega_{s}$ is the synchronous speed, the swing equation is found:

$$
\begin{aligned}
& \frac{2}{p} M \frac{d^{2} \theta_{m}}{d t^{2}}=P_{\text {in }}-P_{\text {out }} \\
& \frac{2 H}{\omega_{s}} \frac{d^{2} \theta_{m}}{d t^{2}}=P_{\text {in }}-P_{\text {out }}
\end{aligned}
$$

And the output voltage can be calculated by:

$$
V_{t R M S}=\sqrt{2} \pi N_{s} \Phi f
$$

where: $N_{s}$ is the number rounds of one-phase windings of the stator; $\Phi$ is the magnetic flux through the stator; $f$ is the system's frequency.

Based on equations (4), (5) and (6), the frequency and voltage response of system and the rotor angle variation of conventional generator can be observed and evaluated.

On the other hand, expanding with a larger system, the stability of the system can be assessed through the equation [9], [10]:

$$
\frac{d}{d t}\left(\frac{1}{2} j_{s} \omega_{s}^{2}\right)=P_{G}-P_{L}
$$


where: $P_{G}$ is the generated power; $P_{L}$ is the load power.

\subsection{Scenarios analysis}

The LSPV penetration level can be determined as the relative total of PV solar power injected into the power grid, which is described by the equation:

$$
\% \text { penetration }=\frac{\text { Total LSPVS capacity }}{\text { Total load power of } D G}
$$

The transient stability analysis is proposed to check the recovery of the grid when different faults occur. Simulations are carried out in two cases, including:

- Case1: Three-phase short-circuit faults on transmission lines with LSPV penetration as Table 2.

- Case 2: LSPV outage according to Table 3.

\section{Simulation result}

\subsection{Case 1}

Table 2. Case 1 - transmission line fault

\begin{tabular}{|c|c|c|}
\hline Scenario & Connected LSPV & Penetration \\
\hline 1 & No LSPV Plants & $0 \%$ \\
\hline 2 & MoDuc PV & $3 \%$ \\
\hline 3 & BinhNguyen PV & $5 \%$ \\
\hline 4 & PhoAn PV & $20 \%$ \\
\hline 5 & Both 3 PVs & $28 \%$ \\
\hline
\end{tabular}

The change of voltage and frequency in the grid is observed in Fig.5 and Fig.6. For the voltage response in Fig.5, with the maximum penetration power of LSPV, the voltage sharply drops but still keeps the value at $35 \%$ when the fault occurs.

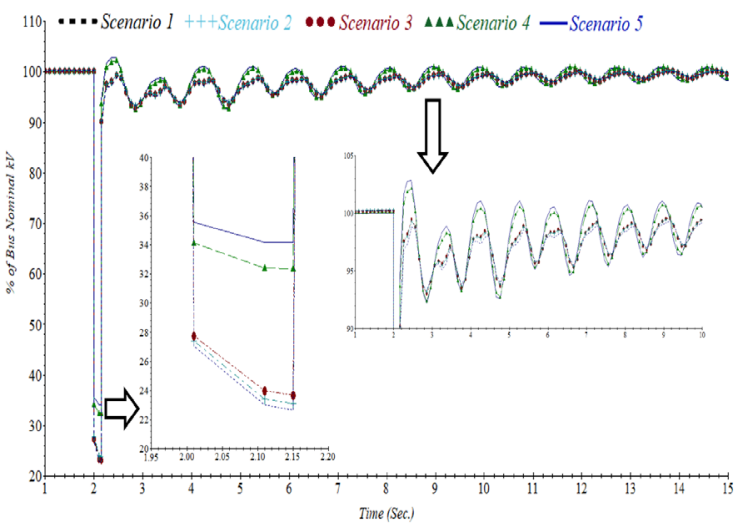

Fig. 5. The voltage response of the grid in case 1.
Meanwhile, without the LSPV connection, the voltage entirely drops to $22 \%$. The remaining scenarios have voltage response changes of $25 \%, 28 \%, 32 \%$ respectively according to increasing levels of the penetration. The frequency response is less fluctuated and recovers faster in Fig.6. Without LSPV connection, the frequency fluctuation is $0.25 \%$, while this rate decreased gradually when the capacity of LSPV connected was $0.12 \%, 0.11 \%, 0.05 \%, 0.04 \%$, respectively.

System inertia increases as the level of LSPV penetration becomes higher, which can be explained from (5) and (6). When LSPVs are connected to the grid, the total generated power increases while the load demand remains constant. As a result, system inertia is improved. As the fault occurs, the large inertia makes the speed of the traditional generators more difficult to change. This effect also leads to the generator terminal voltage being kept more stable in the event of system failures. The high penetration of $\mathrm{PV}$, in this case, contributes to the increase grid stability, when the voltage and frequency are experienced a low level of oscillation and the recovery is faster than one in lower level of PV penetration.

On the other way, the speed and rotor angle of nearby conventional generator is shown in Fig.7 and Fig.8, respectively. The synchronizing speed has lower fluctuations during the transition. LSPV penetrating the grid causes system inertia to increase so that it is difficult for generators to change operating speed. Meanwhile, the rotor deviation angle tends to increase and become more turbulent because of large reactive power transmitted to the fault location for raising the voltage. This change can be explained through (4) and (5).

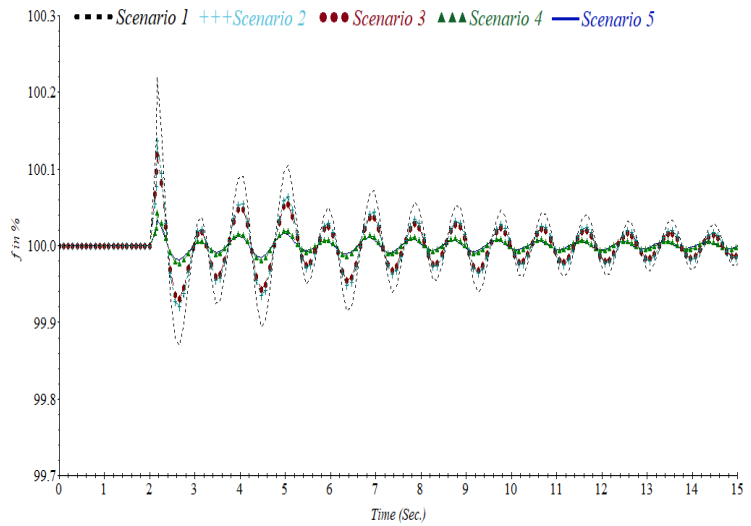

Fig. 6. The frequency response of the grid in case 1 . 


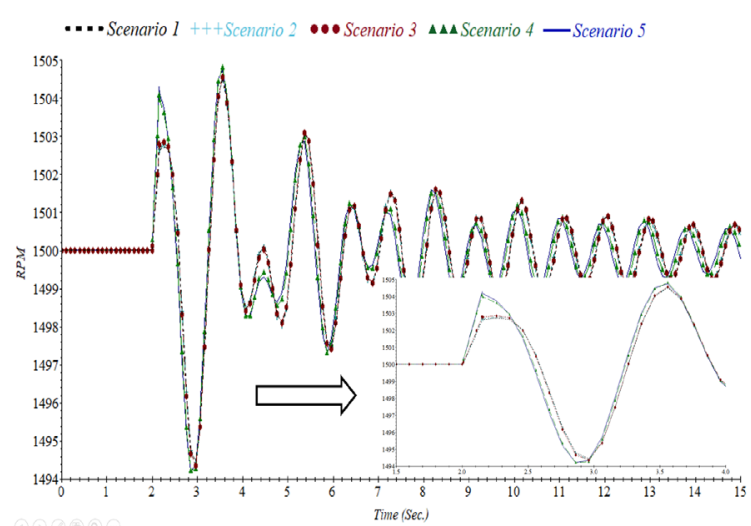

Fig. 7. Generator speed of conventional generator nearby in case 1 .

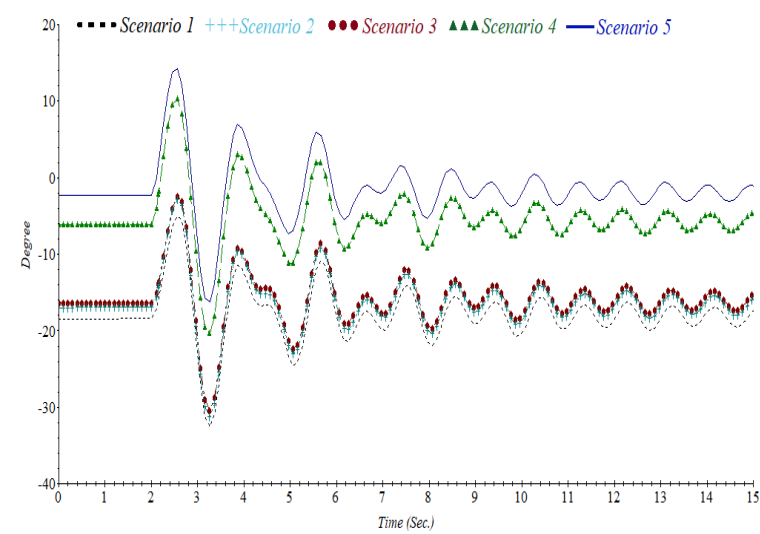

Fig. 8. Rotor angle of conventional generator nearby in case 1

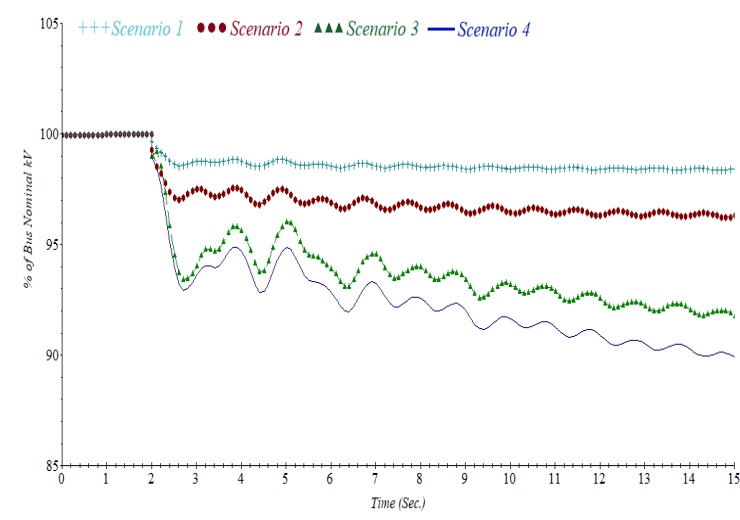

Fig. 9. The voltage response of the grid in case 2 .

\subsection{Case 2}

Because of weather conditions such as cloudy, rainy, etc., the output power of PV can be immediately interrupted. This is the biggest challenge in the

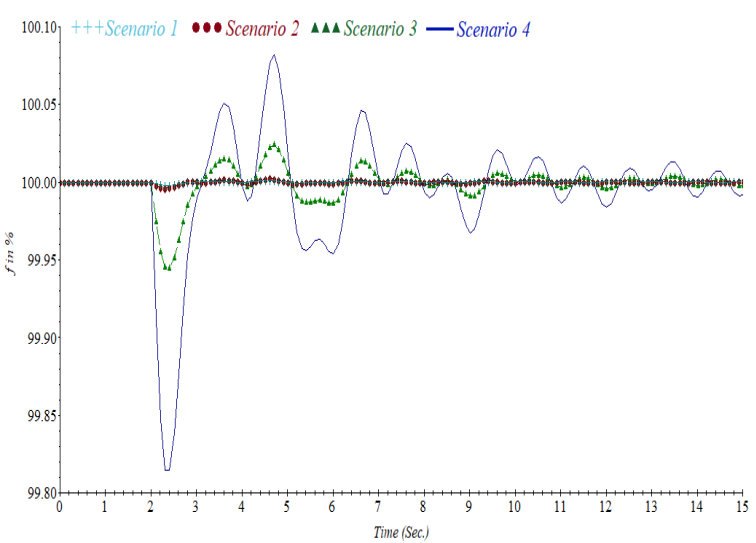

Fig. 10. The frequency response of the grid in case 2

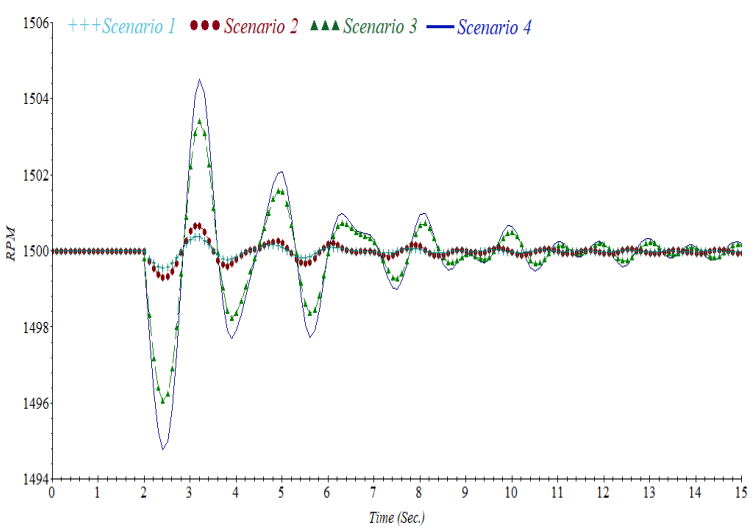

Fig. 11. Generator speed of conventional generator nearby in case 2 .

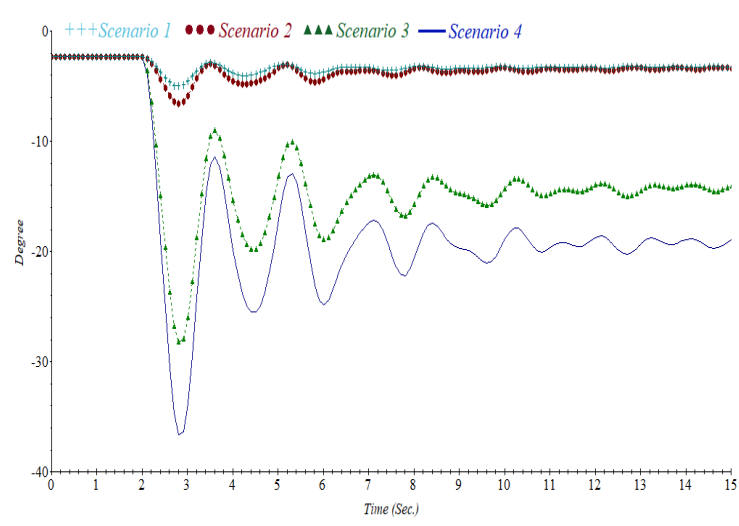

Scenario $1 \bullet \bullet$ Scenario $2 \Delta \mathbf{\Delta}$ Scenario 3 - Scenario 4

Fig. 12. Rotor angle of conventional generator nearby in case 2 .

operation of PV. In this case, all LSPV plants generate full power for the worst scenarios and the same survey work is done in Case 1. The voltage and frequency are shown in Fig.9 and Fig.10 respectively. 
In Fig.9, it can be seen that the voltage suffers more difficulties in the recovery process when too much power is lost from LSPVs. In scenarios 3 and 4, the voltage decreases gradually after the breakdown and drops below $95 \%$, while the other 2 scenarios may show the voltage response allowing stable operation according to the grid code. The frequency responses in Fig.10 are more unstable and have poor recovery when suddenly losing power from the PV. The maximum LSPV penetration scenario shows that the frequency oscillates around $0.2 \%$ as the incident starts, whereas the fluctuation is at around $0.01 \%$ with the lowest penetration level.

Table 3. Case 2 - LSPV outage.

\begin{tabular}{|c|c|c|}
\hline Scenario & LSPV outage & Penetration \\
\hline 1 & MoDuc PV & $3 \%$ \\
\hline 2 & BinhNguyen PV & $5 \%$ \\
\hline 3 & PhoAn PV & $20 \%$ \\
\hline 4 & Both 3 PVs & $28 \%$ \\
\hline
\end{tabular}

The results present the opposite behavior compared to the first case. The small capacity of LSPV does not cause any problems for the grid that can still operate stably in scenarios 1 and 2 after the fault. Meanwhile, more serious incidents when a lot of LSPV output power is interrupted like scenarios 3 and 4 are shown in Table 3 . When too much transmitted power is lost, according to (5) and (6), the frequency will have an aggressive change, causing a large disturbance in the grid. The deviation angle of generators after the occurrence of a strong decrease reduces the reactive power in the grid, leading to difficulty in voltage recovery.

\section{Conclusion}

When the fault occurs outside the plant, the high level of LSPV penetration keeps the frequency and voltage stable. However, the sudden loss of power LSPV plants will threaten the operation of the system. In this case, just the shutdown of PhoAn PV is enough to cause the system to disintegrate if there are no ancillary services.

Therefore, the solution that resolved the changes in voltage and frequency or when the plant suddenly loses all power needs to be studied. The grid must be properly regulated by using a battery system, SVC, STACOM, etc. On the other hand, the optimal hosting capacity of LSPV needs to be conducted in order to be able to plan the number and capacity of LSPV connected to the grid in the future.

\section{Acknowledgments}

This research is funded by Vietnam National Foundation for Science and Technology Development (NAFOSTED) under grant number $102.02-2020.07$.

\section{References}

[1] International Renewable Energy Agency, Renewable Energy Statictis 2020, ISBN: 978-92-9260-246-8, (July 2020) [Online]. Available: https://www.irena.org/publications/2020/Jul/Renewa ble-energy-statistics-2020.

[2] International Renewable Energy Agency, Future of Solar Photovoltaic, ISBN:n978-92-9260-156-0, (Nov. 2019). [Online], Available: https://www.irena.org/publications/2019/Nov/Futureof-Solar-Photovoltaic

[3] M. Q. Duong, N. T. Nam Tran, G. N. Sava and M. Scripcariu, The impacts of distributed generation penetration into the power system, 2017 International Conference on Electromechanical and Power Systems (SIELMEN), Iasi, (2017) pp. 295-301.

[4] Galen L Barbose, U.S. Renewables Portfolio Standards: 2019 Annual Status Update, Lawrence Berkeley National Laboratory, (July 2019). [Online], Available website: https://eta publications.lbl.gov/sites/default/files/rps_annual_sta tus_update-2019_edition.pdf

[5] Y. Liu, J. Bebic, B. Kroposki, J. de Bedout and W. Ren, Distribution System Voltage Performance Analysis for High-Penetration PV, 2008 IEEE Energy 2030 Conference, Atlanta, GA, (2008) pp. 18.

[6] S. S. Refaat, H. Abu-Rub, A. P. Sanfilippo and A. Mohamed, Impact of grid-tied large-scale photovoltaic system on dynamic voltage stability of electric power grids, in IET Renewable Power Generation, vol. 12, no. 2, (2018) pp. 157-164.

[7] Tennet TSO GmbH, Grid Code for High and Extra High Voltage, (2015). [Online], Available online: https://www.tennet.eu/electricitymarket/germancustomers/grid-customers/gridconnection-regulations.

[8] Electricity Regulatory Authority of Vietnam, Circular No. 39/2015/TT-BCT of the Government of Vietnam [Online], Available: http://www.erav.vn.

[9] J. Duncan Glover, Thomas J. Overbye, Mulukutla S. Sarma, Power System Analysis \& Design, sixth edition, Cengage Learning, ISBN: 978-1-305-632134, (2015).

[10] U. Andreas, T.S. Borsche and G. Andersson, Impact of Low Rotational Inertia on Power System Stability and Operation, IFAC World Congress 2014, Capetown, South Africa, (2014). 